\title{
1 Determinants of the difficulty of leadless pacemaker implantation
}

2 Christophe Garweg, $\mathrm{MD}^{1,2}$, Bert Vandenberk, $\mathrm{MD}, \mathrm{PhD}^{1,2}$, Stefaan Foulon ${ }^{2}$, Patrick Hermans ${ }^{2}$, 3 Patricia Poels ${ }^{2}$, Peter Haemers $\mathrm{MD}, \mathrm{PhD}^{1,2}$ Joris Ector $\mathrm{MD}, \mathrm{PhD}^{1,2}$ and Rik Willems, $\mathrm{MD}, \mathrm{PhD}{ }^{1,2}$

5 1. University of Leuven, Department of Cardiovascular Sciences

6 2. University Hospitals Leuven, Cardiology

8 Short title: Predictors of leadless pacemaker implantation.

\section{Corresponding author:}

10 Christophe Garweg, M.D., Department of Cardiovascular Sciences, Herestraat 49, 3000 Leuven, 11 Belgium

12 Tel: +3216342079

Email: christophe.garweg@uzleuven.be

14 Word count: 2906 (including the abstract). Abstract only: 249 words

Contributors: All the authors contributed significantly to the completion of the study and the manuscript, including reading and approval of the manuscript in its current form.

Potential conflicts of Interest: RW/JE/CG research funding: Biotronik, Boston Scientific,

18 Medtronic. Speakers and consultancy fees: Medtronic, Boston Scientific, Biotronik, St Jude

19 Medical, Sorin. CG was supported as predoctoral clinical researcher by the Fund for Scientific 
Background: The Micra Transcatheter Pacing System is implanted directly in the right ventricle (RV) through the femoral vein using a steerable transcatheter delivery system. The present study was done to identify determinants of difficult leadless pacemaker implant procedures including operator, patient and RV anatomical characteristics.

Methods: All patients who underwent a Micra implant from July 2015 to December 2018 at our centre were analysed. From an RV angiogram acquired during implantation, RV geometry including systolic and diastolic volumes and ejection fraction was characterised. The presence of septo-marginal trabeculation was noted.

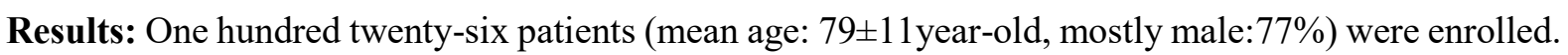
Mean Micra RV implant procedure time was $24 \pm 23$ min, with $1.7 \pm 1.3$ deployments of the device. No significant change in implant procedure time was observed after the first 30 implants. Eleven patients had a prominent septal component of the septo-marginal trabeculation in the RV. Univariate analysis showed that the procedure time was positively correlated with the presence of a prominent septal component of the septo-marginal trabeculation $(\mathrm{p}<0.001)$ or an episode of heart failure $(\mathrm{p}=0.02)$ and negatively correlated with the number of procedures performed by the operator $(\mathrm{p}<0.001)$. After multivariable analysis, only the presence of a prominent septal component of the septo-marginal trabeculation $(p<0.001)$ and the number of procedures performed by the operator $(p<0.001)$ were associated with the implant procedure time.

Conclusions: In our experience, implant procedure time of a Micra leadless pacemaker depended on the presence of a prominent septal component of the septo-marginal trabeculation and operator experience.

Keywords: Micra TPS, leadless pacemaker, right ventricle, anatomy 
Pacing therapy is the cornerstone for the treatment of symptomatic bradycardia and high degree atrioventricular block ${ }^{1}$. Despite substantial continued evolution of conventional pacing system for almost 60 years, transvenous leads and the presence of a subcutaneous generator are still the Achilles heel of the technology. Vascular damages, pneumothorax, lead failure, infections, skin erosion and hematomas remain major issues associated with transvenous pacing ${ }^{2}$.

Leadless pacemakers were developed to reduce the complication rate associated with traditional transvenous devices. Micra TPS (Transcatheter Pacing system, Medtronic, Minneapolis, MN, US) is currently the only available leadless pacemaker. The device is delivered through a femoral vein using a 23 French deflectable delivery catheter and is anchored in the right ventricle (RV) by four nitinol tines

${ }^{3}$. Real-world data demonstrated a high rate of successful implant procedure $(99.1 \%)$ and was associated with a low number of major complications $(2.7 \% \text { at } 12 \text { month follow-up })^{4}$. Despite these excellent results, it remains unclear what the determinants of a difficult implantation procedure (such as increased procedure time or required redeployments) are. Procedure time and the number of deployments could depend on operator, patient demographics and RV anatomy. New technologies and procedures have a learning curve where procedure times may decrease as an operator gains experience. For example, the procedure duration for atrial fibrillation ablation continues to fall past 100 procedures while the procedure times and complications rate for transcatheter aortic valve replacement decreases significantly after \pm 25 procedures $^{5,6}$. The anatomy of the heart may also influence the implant procedure as seen in ablation of atrial flutter or implantation of a left ventricular lead for cardiac resynchronization therapy ${ }^{7,8}$. We have previously shown that total duration of leadless pacemaker implant procedure increased due to complex peripheral venous anatomy including tortuosity, stenosis and occlusion 9 . It is unknown whether the time needed to implant a leadless pacemaker in the RV, socalled "right ventricular implant time", defined as the time from transcatheter delivery of the device in 
evaluated the determinants of the RV procedure duration of a Micra leadless pacemaker including operator experience and RV anatomical characteristics in a large single centre experience.

\section{Methods}

\section{Study population and implant procedure}

Patients 18 years and older with class I or II guideline recommendations for ventricular pacing who underwent a Micra TPS implant procedure at the University Hospitals of Leuven between July 2015 and December 2018 were included in this retrospective study ${ }^{1}$. All procedures were performed by one single operator (CG). All patients were included in a prospective monocentric registry. Baseline demographics, clinical and procedural characteristics were recorded prospectively for all patients and analysed retrospectively. The implant procedure was performed as previously described ${ }^{3,9}$. A right ventriculogram was performed in patients to assess RV anatomy and to guide device positioning except in the presence of severe renal insufficiency (GFR $<30 \mathrm{ml} / \mathrm{min}$ ) or iodine contrast allergy. A pigtail catheter was inserted via a femoral vein into the RV and iodine contrast agent diluted to $50 \%$ (a total volume of $60 \mathrm{~mL}$ ) was injected at a rate of $15 \mathrm{~mL} / \mathrm{s}$. Cardiac angiography was performed simultaneously from two directions $\left(30^{\circ}\right.$ right anterior oblique $[\mathrm{RAO}]$ and $40^{\circ}$ left anterior oblique [LAO] views with biplane cardiac digital angiography system (Siemens Axiom Artis dBC fluoroscopy system, Erlangen, Germany). In the beginning of the experience, an RV angiogram was only performed if felt necessary by the operator, but from the $20^{\text {th }}$ implant procedure, it was systematically performed unless contraindicated. Micra implantation was performed while integrating end-diastolic ventriculography images with biplane fluoroscopy.

\section{Angiography derived RV anatomy}

RV end-diastolic volume (RVEDV) and end-systolic volume (RVESV) were measured on right ventriculography as previously described ${ }^{10}$. RV ejection fraction (RVEF) was calculated by subtracting RVESV from RVEDV and dividing the difference by RVEDV. The following RV dimensions were measured on the RAO $30^{\circ}$ incidence view (Figure 1) to characterize the RV anatomy: (1) length of 
tricuspid valve annulus, (2) the distance between the middle of the tricuspid valve annulus and the RV apex, (3) the length of RV base (distance from the inferior insertion of the tricuspid valve to the RV apex), (4) the length of RV anterior wall, and (5) the angle between those two anatomical landmarks. The presence of a prominent septal component of the septo-marginal trabeculation was systematically reported based on anonymized angiographic images judged by two independent investigators (CG and SF) (Figure 2). In case of disagreement, both investigators openly discussed the angiographic image and came to a final conclusion.

\section{Post-Implant follow-up}

All patients were monitored for 24 hours after the procedure. A transthoracic echocardiogram was obtained the day after implantation to exclude the presence of any pericardial effusion and to measure left ventricular end-diastolic diameter (LVEDD) and LV ejection fraction (LVEF). Device electrical performance (pacing threshold at $0.24 \mathrm{~ms}$, R-wave amplitude and impedance) was controlled at implant, discharge, $1,6,12,18$ and 24 months after implantation.

\section{Statistical analysis}

Normally distributed continuous parameters are presented as mean \pm standard deviation (SD), nominal data are presented as numbers with percentages. Groups were compared using the Student's t-test or one-way ANOVA with a Tukey post-hoc test when appropriate. The presence of a prominent septal component of the septo-marginal trabeculation by 2 independent observers was compared using Cohen's kappa coefficient and the percentage agreement. Univariate linear regression analysis for RV implant time was performed. Subsequently, multivariate linear regression analysis was performed (forward method), including all variables with a univariate p-value $<0.100$. In all models, a constant was included and the coefficient B with its $95 \%$ confidence interval is reported. To characterize the learning curve, implants were grouped into quintiles of 30 patients. The RV implant time was compared using repeated measures ANOVA with a Tukey post-hoc multiple comparison test. All statistical analyses were performed using SPSS (IBM statistics, version 25). 


\section{Results}

125 From July 2015 to December 2018, 125 out 126 patients (99.2\%) who were successfully implanted with

126 a Micra TPS were included in this analysis. One implant procedure $\left(18^{\text {th }}\right)$ was not successful within one patient with an athlete's heart and highly dilated RV. Demographical characteristics of the population are summarized in Table 1. Patients were $79 \pm 11$ year old, mostly male (77\%) and had a history of atrial fibrillation (65\%). The mean Micra RV implant procedure time was 24 \pm 23 min (range 5-109), with $1.7 \pm 1.3$ (range 1-9) deployments per procedure.

There were no device dislodgement nor pericardial effusion. During follow up, two major complications (1.6\%) were reported. Loss of pacing capture was observed in the first 6 month post-implant in two patients with a prominent septal component of the septo-marginal trabeculation $\left(4^{\text {th }}\right.$ and $80^{\text {th }}$ implant procedure). The device was repositioned during a scheduled combined mitral and tricuspid annuloplasty for the $4^{\text {th }}$ patient and a second device was implanted in the $80^{\text {th }}$ patient (without extraction of the first one) ${ }^{11}$. After re-implantation, no further electrical issues were encountered. Except for these both patients, electrical parameters remained stable during the follow-up independently of the presence of a prominent septal component of the septo-marginal trabeculation.

The minor complications rate was $4.8 \%: 2$ severe vagal reaction during the implant procedure in $17^{\text {th }}$ and $56^{\text {th }}$ patients, 2 events at the groin puncture site $\left(1\right.$ minor hematoma $\left[88^{\text {th }}\right.$ patient $]$ and 1 asymptomatic arteriovenous fistula with spontaneous healing within 4 weeks $\left[22^{\text {nd }}\right.$ patient $]$ ) and 2 deep venous thrombosis within the first month after the implant procedure in $42^{\text {nd }}$ and $81^{\text {st }}$ patients).

Table 1 summarizes the angiographically derived anatomical characteristics of the RV available for 109 patients where an RV angiogram was performed. There were large inter-individual variations of the measured RV dimensions and volumes (RVESV and RVEDV). Mean RV ejection fraction was $52 \pm 9$ $\%$. Angiographic images demonstrated the presence of a prominent septal component of the septomarginal trabeculation along the interventricular septum in 11 patients $(10.1 \%)$ (Figure 2). Figure 3 shows anatomical representation of a right ventricle without (panel A) and with (panel B) the presence 
of prominent septal component of the septo-marginal trabeculation along with positioning of a Micra pacemaker. Presence of this anatomical feature significantly lengthened the Micra RV implant procedure: $60.6 \pm 37.4 \mathrm{~min}$ versus $18.8 \pm 17.3 \mathrm{~min}(\mathrm{p}<0.001)$ (Figure 4).

RV implant procedure time was correlated with the operator experience $(p<0.001)$. A stable RV procedure time was reached after the first 30 procedures, as shown in Figure 4. Mean RV procedure time was $46.2 \pm 31.9 \mathrm{~min}$ (range: $12-131 \mathrm{~min}$ ) for the first 30 procedures decreasing significantly to $22.1 \pm 14.4 \mathrm{~min}$ (range: $12-72 \mathrm{~min}$ ) for the next 30 procedures $(\mathrm{p}<0.001)$. Subsequently, no statistically significant difference was observed among the quintiles (procedures 61-90: 17.5 \pm 12.2 min (range: 550); procedures 91-120: 13.2 $\pm 12.1 \mathrm{~min}$ (range 5-79); procedures 121-126: 13.1 \pm 10.9 min (range: 7 40)). The same evolution was observed in the Micra TPS total procedure time (Figure 5).

In a univariate analysis, the RV implant procedure time was significantly correlated with a previous episode of heart failure ( $\mathrm{p}=0.022$ ), the presence of a prominent septal component of the septo-marginal trabeculation along the interventricular septum $(\mathrm{p}<0.001)$ and the number of procedures performed by the operator $(\mathrm{p}<0.001)$. In multivariable analysis, the presence of a prominent septal component of the septo-marginal trabeculation along the interventricular septum $(\mathrm{p}<0.001)$ and the number of procedures performed by the operator were the only parameters significantly associated with the implant procedure duration.

The RV implant time was correlated with the number of device redeployments $(p<0.001)$ (Figure 6). The number of redeployments was similarly correlated to the presence of a prominent septal component of the septo-marginal trabeculation and operator experience. In patients with an absence of prominent septal component of the septo-marginal trabeculation, the median number of redeployments was 0 (IQR 0 - 1) versus 1 (IQR 0 - 4) in patients with a prominent septal component of the septo-marginal trabeculation $(\mathrm{p}=0.004)$. The number of device redeployment decreased with the operator experience $(\mathrm{p}=0.045)($ Figure 7).

\section{Discussion}


There are two main findings in this study. First, the presence of a prominent septal component of the septo-marginal trabeculation along the interventricular septum was the only RV anatomical parameter that conditioned the RV implant time. Secondly, the influence of operator experience on implant procedure duration reached steady state after approximately 30 procedures.

To the best of our knowledge, no previous studies have considered the potential impact of RV anatomy on the implant procedure of leadless pacemakers. The use of RV angiography is not mandatory for the implantation of leadless pacemakers, however, its use in our implant protocol from the beginning of our experience has contributed to clearly define the RV anatomy without any negative impact on the safety of the procedure. We did not observe any significant impairment of the renal function since contrast use was avoided in patients with severe renal dysfunction. In our population, we showed that the presence of a prominent septal component of the septo-marginal trabeculation along the interventricular septum increased procedural difficulty as reflected in a longer implant procedure time. From our results, we can speculate that the difficulty in reaching low septal or apical positions during an implant procedure requiring implantation in a higher septal position may be related to the presence of a prominent septal component of the septo-marginal trabeculation. Anatomical studies have described and classified the septo-marginal trabeculation in humans ${ }^{12-14}$. Bandeira et al. considered that the septo-marginal trabeculation presented two components: a septal component along the interventricular septum and a septal-papillary component extending from the septum to the anterior papillary muscle. According to their observations, the septal component could be either seen macroscopically forming a third-order fleshy column (with a prominent septal portion) or was visible only by means of dissection (without a prominent septal portion) ${ }^{14}$. Their description is in accordance with our angiographic images of the septo-marginal trabeculation with a septal component visible only in some patients. We believe that the presence of a fleshy column along the interventricular septum contributes to a reduction in the ability to rotate clockwise the steerable catheter to reach straightforward a septal implant position, therefore lengthening the duration of the implant procedure. Interestingly, loss of pacing capture observed during follow-up occurred in 2 patients with a prominent septal component of the septo-marginal trabeculation that might have prohibited the adequate anchorage of 
the device in the myocardial wall. The design of the study does not permit to identify the presence of a prominent septo-marginal trabeculation before the implant procedure. Nevertheless, a peri-operative RV angiogram may be helpful to identify a prominent trabeculation and therefore suggest a higher septal position for device implantation. This should be evaluated in a prospective study.

Secondly, we observed that procedure duration decreased with experience of the operator. The learning curve of the operator was associated with an initial high rate of success for implantation and no excess of complications. In our study, the procedure time for leadless pacemaker implantation performed by one single operator (CG) significantly decreased from the first 30 procedures without any subsequent significant reduction afterwards. This illustrates the importance of a large volume of procedures by a single operator needed to gain experience in leadless pacemaker implantation. Our study protocol allows for the first time to assess the number of procedures needed to reach a "steady-state implant procedural duration". Previously, El Chami et al. reporting on the data of the Micra Transcatheter Pacing Study also showed that the procedure duration decreased with implant number and that the complication rate did not seemed to be associated with case number. The analysis included 720 successful procedures performed by 94 operators with a median of four implantations (range 1-55). The procedure time decreased from the first quartile ( 2 first procedures: $42.7 \pm 29.9 \mathrm{~min}$ ) to the fourth quartile (>12 implants: $25.9 \pm 13 \mathrm{~min}$ ) representing a reduction by $2 \%$ from the prior procedure with each subsequent implant $(95 \% \text { ci: } 0.7-3.2 \%, \mathrm{p}=0.002)^{15}$. However, due to the design of the study protocol, the number of procedures needed to achieve optimal procedure performance could not be assessed. In that study, the definition of procedure time (time in minutes from introducer in to out) differed from ours which was the time from transcatheter delivery of the device in the right atrium and retrieval of the tether. Procedural time for the Nanostim leadless pacemaker that uses another modality for delivery in the RV compared to the Micra TPS also showed a clear learning curve. In a cohort of 1439 patients implanted by 171 operators, procedure duration defined as time from venous access to removal of the introducer sheath also significantly decreased from the first quartile (first 2 implants: $30.9 \pm 19.1 \mathrm{~min}$ ) to the fourth quartiles (> 10 implants: $21.6 \pm 13.2 \mathrm{~min})^{16}$. 
227 Since the complication rate was low from the start of our experience, the number of device 228 redeployments associated with the procedure duration also allowed assessment of the efficiency of the 229 procedure. Our data showed that the requirement for device redeployment decreased with operator's experience. This was also observed by El-Chami et al. for the Micra and by Tjong et al. for the Nanostim ${ }^{15,16}$. Furthermore, we showed that the need for device redeployment was correlated with the presence of prominent septo-marginal trabeculation.

\section{Limitations:}

This study has limitations. First, we reported the experience of a single operator. However, this approach represented a unique way to assess the number of implant procedures required to reach a steady state in procedural time. Fluoroscopy time that was reported for the procedure from venous access to retrieval of the catheter sheath in our database could not be correlated with the procedure time as we defined it. Nevertheless, we were mainly interested to determine the time required to anchor the device depending on RV anatomy. Therefore, we excluded the time interval needed to navigate from the femoral puncture point to the right atrium that is highly dependent on venous anatomy ${ }^{9}$.

\section{Conclusion}

242 Except for the presence of prominent septal component of the septo-marginal trabeculation in the RV,

243 the implant procedural duration of a leadless pacemaker Micra TPS and number of device deployments 244 depended on the operator's experience. No further significant decrease of the implant procedure time 245 was observed after approximately 30 procedures.

\section{Authors contributions:}

247 C. Garweg elaborated the concept/design of the study, performed the data analysis/interpretation and 248 wrote the present manuscript. B. Vandenberk performed the statistical analysis and reviewed the article.

249 S. Foulon contributed for the analysis of the data. P. Poels and P. Hermans collected the data. P. Hamers 250 and J. Ector contributed for the elaboration of the study design and reviewed the manuscript. R. Willems 
contributed for the elaboration of the study design, data analysis/interpretation and reviewed the manuscript. Finally, all authors read and approved the manuscript in its current form.

\section{Acknowledgements}

We thank Vince Splett of Medtronic for assistance in the preparation of this manuscript.

\section{References:}

1. European Society of C, European Heart Rhythm A, Brignole M, et al. 2013 ESC guidelines on cardiac pacing and cardiac resynchronization therapy: the task force on cardiac pacing and resynchronization therapy of the European Society of Cardiology (ESC). Developed in collaboration with the European Heart Rhythm Association (EHRA). Europace. 2013;15(8):1070-1118.

2. Udo EO, Zuithoff NP, van Hemel NM, et al. Incidence and predictors of short- and longterm complications in pacemaker therapy: the FOLLOWPACE study. Heart Rhythm. 2012;9(5):728-735.

3. Ritter P, Duray GZ, Steinwender C, et al. Early performance of a miniaturized leadless cardiac pacemaker: the Micra Transcatheter Pacing Study. Eur Heart J. 2015;36(37):2510-2519.

4. El-Chami MF, Al-Samadi F, Clementy $\mathrm{N}$, et al. Updated performance of the Micra transcatheter pacemaker in the real-world setting: A comparison to the investigational study and a transvenous historical control. Heart Rhythm. 2018.

5. Cappato R, Calkins $\mathrm{H}$, Chen SA, et al. Updated worldwide survey on the methods, efficacy, and safety of catheter ablation for human atrial fibrillation. Circ Arrhythm Electrophysiol. 2010;3(1):32-38.

6. Alli O, Rihal CS, Suri RM, et al. Learning curves for transfemoral transcatheter aortic valve replacement in the PARTNER-I trial: Technical performance. Catheter Cardiovasc Interv. 2016;87(1):154-162.

7. Gamble JHP, Herring N, Ginks M, Rajappan K, Bashir Y, Betts TR. Procedural Success of Left Ventricular Lead Placement for Cardiac Resynchronization Therapy: A MetaAnalysis. JACC Clin Electrophysiol. 2016;2(1):69-77.

8. Baccillieri MS, Rizzo S, De Gaspari M, et al. Anatomy of the cavotricuspid isthmus for radiofrequency ablation in typical atrial flutter. Heart Rhythm. 2019.

9. Garweg C, Ector J, Voros G, et al. Monocentric experience of leadless pacing with focus on challenging cases for conventional pacemaker. Acta Cardiol. 2017:1-10. 
10. Wellnhofer E, Ewert $P$, Hug J, et al. Evaluation of new software for angiographic determination of right ventricular volumes. Int J Cardiovasc Imaging. 2005;21(6):575585.

11. Garweg C, Ector J, Oosterlinck W, Willems R, Herijgers P. Successful repositioning of leadless cardiac pacemaker during open heart surgery. Acta Cardiol. 2017;72(4):503504.

12. Brown M. Construction of the Ventricles in the Mammalian Heart. J Anat Physiol. 1889;23(Pt 2):250-255.

13. Depreux $\mathrm{R}$, Mestdagh $\mathrm{H}$, Houcke M. [Comparative morphology of the trabecula septomarginalis in terrestrial mammals]. Anat Anz. 1976;139(1-2):24-35.

14. Bandeira ST, Wafae GC, Ruiz C, Nascimento SR, Fernandes JR, Wafae N. Morphological classification of the septomarginal trabecula in humans. Folia Morphol (Warsz). 2011;70(4):300-304.

15. El-Chami M, Kowal RC, Soejima K, et al. Impact of operator experience and training strategy on procedural outcomes with leadless pacing: Insights from the Micra Transcatheter Pacing Study. Pacing Clin Electrophysiol. 2017;40(7):834-842.

16. Tjong FVY, Beurskens NEG, Neuzil $P$, et al. The learning curve associated with the implantation of the Nanostim leadless pacemaker. J Interv Card Electrophysiol. 2018;53(2):239-247. 
Figure 1: RV angiogram in RAO view $30^{\circ}$ : RV dimensions were measured at the end of diastolic phase: length of the tricuspid annulus (1), distance between the middle of the tricuspid valve annulus and the RV apex (2), length of RV base (distance from the inferior insertion of the tricuspid valve to the RV apex (3), length of RV anterior wall (4), and angle between those two anatomical landmarks (5).

Figure 2: RV angiogram in RAO view $30^{\circ}$. A. Example of a patient with RV prominent septo-marginal trabeculation (black arrows).

B. Example of a patient without RV prominent septo-marginal trabeculation.

Figure 3: Segmental anatomy of the RV in RAO view. A. In the absence of a prominent septal component of the septo-marginal trabeculation, the leadless pacemaker can be easily implanted on several septal positions. B. The presence of prominent septal component of the septo-marginal trabeculation $(*)$ prevents to reach easily an apico-septal or mid to low septal position. In this case, targeting the high septum should be preferred.

Figure 4: Micra TPS RV implant time related to the presence or the absence of a prominent septomarginal trabeculation.

Figure 5: Micra TPS RV implant time and Micra TPS total procedure time (min) divided in 5 quintiles of 30 procedures. The steady state was reached after 30 procedures $(\mathrm{p}<0.001)$.

Figure 6: Correlation of the number of device redeployments with the Micra TPS RV implant time.

Figure 7: Evolution of number of device redeployment with operator experience. 